\title{
Amount Recovered Infinity Observed
}

National Cancer Institute

\section{Source}

National Cancer Institute. Amount Recovered Infinity Observed. NCI Thesaurus. Code C112032.

The cumulative amount recovered extrapolated to infinity, calculated using the observed value of the last non-zero concentration. 